\title{
ADDITIONS AND UPDATED NAMES FOR GRASSES OF DURANGO, MEXICO
}

\author{
Yolanda HerRera-Arrieta \\ Instituto Politécnico Nacional, Centro Interdisciplinario de Investigación \\ para el Desarrollo Integral Regional, Unidad Durango, Sigma 119, \\ Fracc. 20 de Noviembre II, 34220 Durango, Durango, Mexico. \\ yherrera@ipn.mx
}

\begin{abstract}
Twelve years after publication of the floristic treatment "Las Gramíneas de Durango", a list of accepted names is updated based on recent botanical work in Durango, Mexico. Ninety-one genera, 367 species, 15 subspecies, 55 varieties and 2 forms are included. Of these, 14 genera are newly recognized for Durango, whereas 17 genera previously reported from the state are now treated as synonyms, and three genera previously recorded are excluded. At the specific and infraspecific levels, 46 changes for synonymy occurred. Fifteen species were excluded, while 43 species and 27 infraspecific taxa were added. Four species are reported as new records for the state.
\end{abstract}

Key words: additions, Durango, grasses, Mexico.

\section{RESUMEN}

Después de 12 años que fuera publicado el tratamiento florístico de "Las gramíneas de Durango" se presenta una relación de nombres aceptados, actualizados con base en trabajos botánicos recientes, de las gramíneas de Durango, México. Se reconocen un total de 91 géneros, 367 especies, 15 subespecies, 55 variedades y 2 formas. De ellos, 14 géneros representan nuevos registros para la entidad, mientras que 17 previamente reconocidos corresponden a sinónimos en el inventario actual y se excluyen tres registrados anteriormente. En los niveles específico e infraespecífico, se realizaron 46 cambios por sinonimia. Quince especies se excluyeron, mientras que 43 y 27 taxa infraespecíficos se adicionaron. Cuatro especies resultaron ser nuevos registros para el estado. 
Palabras clave: adiciones, Durango, gramíneas, México.

\section{INTRODUCTION}

Because of the great economic importance and large size of the family, the nomenclature of Poaceae is huge and elaborate. The first comprehensive treatment of grasses from North America was prepared by Beal (1896) and later Hitchcock (1913) prepared a treatment of Mexican grasses. In addition to making plant collections throughout the continent since the end of the 19th Century, American agrostologists continued to verify the identity of the taxa, as well as describe and apply names to new species. As a result of their efforts, a number of important floristic and floristic-ecologic reports from large regions have been published, e.g., North America (Hitchcock, 1935 and Hitchcock \& Chase, 1951), "Nueva Galicia" (McVaugh, 1983), and "The grasslands of Durango" (Gentry, 1957). Most taxa have more than one name, because they were described by different authors, and some names have been repeatedly misapplied to different species. In both cases, valid names since have been established with the later names relegated to synonymy.

The first important catalogue of grasses in the New World was the "Index of American grasses", edited by Chase and Niles (1962), who included all accepted names and synonymy known at the time. Another very recent compilation of this kind is the Catalogue of New World Grasses (Soreng et al. 2013, http://www.tropicos.org/Project/ CNWG, continually updated) with hard copies published by subfamilies (Judziewicz et al. 2000, Peterson et al. 2001, Zuloaga et al. 2003, and Soreng et al. 2003, 2013). Dávila et al. (2006) compiled and edited the corresponding data of the accepted names and synonymy for the Mexican taxa in the "Catálogo de las Gramíneas de México". The present article is based on these two last catalogues, and has been further enriched by recently published treatments (e.g., Finot et al. 2004, Bess et al. 2006, Zuloaga et al. 2007, Bell and Columbus 2008, Valdés-Reyna et al. 2009, Columbus and Smith 2010, Peterson et al. 2010, Chemisquy et al. 2010, Peterson et al. 2012) and/or additions of new described species (Finot et al. 2004, Peterson et al. 2004, 2006, Peterson and Columbus 2009). In addition, I have included new reports based on review of recently collected grass specimens in the state of Durango (Herrera Arrieta et al. 2004, Herrera Arrieta and Cortés Ortíz 2009, 2010, Herrera Arrieta et al. 2012).

In conjunction with projects that the Mexican Comisión Nacional para el Conocimiento y Uso de la Biodiversidad (CONABIO) has funded to pursue biodiversity research, I have found that there are substantial changes that should be 
reported to the community of agrostologists, grassland students, and general users. These include newly found taxa, new synonyms, and corrections to erroneous determinations. The purpose of the current study is to review these changes and provide an updated list of the names of the currently accepted taxa for Durango.

\section{MATERIALS AND METHODS}

Grass specimens from Durango are constantly being deposited by scientists and students at the CIIDIR herbarium, and this material has been the source of new records during the past 12 years. In addition, other important herbaria with many Mexican collections were examined: CHAPA, ENCB, HUAA, IEB, MEXU, SLPM, TAES, US. Most of the synonyms and nomenclatural changes follow those given in the Catalogue of New World Grasses (Soreng et al. 2013, http: //www.tropicos.org).

\section{RESULTS}

Tables 1 and 2 show the numerous modifications at the generic, specific or infraspecific level for grasses in Durango that have occurred during the last 12 years. Accepted taxa are displayed in bold.

In summary, an additional 15 genera are currently recognized as occurring in Durango (these indicated by * in Table 1 and 2), whereas 17 genera previously recognized for the state are now treated as synonyms (**Table 1). Three previously recorded genera are excluded: Allolepis Soderstr. \& H.F. Decker, Guadua Kunth and Setariopsis Scribn. Guadua was based on an erroneous determination; Allolepis and Setariopsis were cited for Durango but a specimen was never found.

Forty-six changes due to synonymy occurred at the specific and infraspecific level (Table 1).

I excluded the following 15 species because they were based on misidentifications: Agrostis alba L. (synonym of Poa nemoralis L.), Allolepis texana (Vasey) Soderstr. \& H.F. Decker, Aristida purpurea var. parishii (Hitchc.) Allred, Axonopus compressus (Sw.) P. Beauv., Bouteloua disticha (Kunth) Benth., Elymus interruptus Buckley, Eragrostis obtusiflora (E. Fourn.) Scribn., Festuca rubra L., Guadua longifolia (E. Fourn.) R.W. Pohl, Hordeum arizonicum Covas, Leptoloma cognata (Schult.) Chase, Panicum virgatum L., Setariopsis latiglumis (Vasey) Scribn., Sorghastrum brunneum Swallen, Urochloa texana (Buckley) R.D. Webster. 
Table 1. Poaceae with nomenclatural changes. $i$ ) Species now considered synonyms of other species (either staying in the same genus or transferred to a different one). ii) Species for which the current recognized genus was segregated from another. iii) Species for which the previously recognized genus was synonymized under another. * New genera recognized as occurring in Durango. **Genera in synonymy.

\begin{tabular}{|c|c|}
\hline Accepted name & Old name (synonym) \\
\hline $\begin{array}{l}\text { ii)*Achnatherum eminens (Cav.) } \\
\text { Barkworth }\end{array}$ & **Stipa eminens Cav. \\
\hline $\begin{array}{l}\text { i) Arundinella hispida (Humb. \& Bonpl. ex } \\
\text { Willd.) Kuntze }\end{array}$ & Arundinella palmeri Vasey ex Beal \\
\hline $\begin{array}{l}\text { iii) Bouteloua dactyloides (Nutt.) } \\
\text { Columbus }\end{array}$ & **Buchloë dactyloides (Nutt.) Engelm. \\
\hline iii) Bouteloua reederorum Columbus & $\begin{array}{l}* * \text { Cyclostachya stolonifera (Scribn.) } \\
\text { Reeder \& Reeder }\end{array}$ \\
\hline $\begin{array}{l}\text { iii) Bouteloua polymorpha (E. Fourn.) } \\
\text { Columbus }\end{array}$ & $\begin{array}{l}\text { **Pentarrhaphis polymorpha (E. Fourn.) } \\
\text { Griffiths }\end{array}$ \\
\hline $\begin{array}{l}\text { iii) Cenchrus clandestinus (Hochst. \& } \\
\text { Chiov.) Morrone }\end{array}$ & $\begin{array}{l}\text { **Pennisetum clandestinum Hochst. \& } \\
\text { Chiov. }\end{array}$ \\
\hline $\begin{array}{l}\text { i) Cenchrus michoacanus H.F. Gut. \& } \\
\text { Morrone }\end{array}$ & Pennisetum crinitum (Kunth) Spreng. \\
\hline i) Cenchrus spinifex Cav. & Cenchrus incertus M.A. Curtis \\
\hline i) Cenchrus longisetus M.C. Johnst. & Pennisetum villosum R. Br. ex Fresen. \\
\hline $\begin{array}{l}\text { ii)*Chascolytrum subaristatum (Lam.) } \\
\text { Desv. }\end{array}$ & **Briza subaristata Lam. \\
\hline $\begin{array}{l}\text { ii) Dinebra panicea subsp. mucronata } \\
\text { (Michx.) P.M. Peterson \& N. Snow }\end{array}$ & Leptochloa mucronata (Michx.) Kunth \\
\hline $\begin{array}{l}\text { ii) *Diplachne fusca subsp. fascicularis } \\
\text { (Lam.) P.M. Peterson \& N. Snow }\end{array}$ & Leptochloa fascicularis (Lam.) A. Gray \\
\hline $\begin{array}{l}\text { ii) Diplachne fusca subsp. uninervia (J. } \\
\text { Presl) P.M. Peterson \& N. Snow }\end{array}$ & $\begin{array}{l}\text { Leptochloa uninervia (J. Presl) Hitchc. \& } \\
\text { Chase }\end{array}$ \\
\hline $\begin{array}{l}\text { ii)*Disakisperma dubium (Kunth) P.M. } \\
\text { Peterson }\end{array}$ & Leptochloa dubia (Kunth) Nees \& N. Snow \\
\hline $\begin{array}{l}\text { ii) Distichlis eludens (Soderstr. \& H.F. } \\
\text { Decker) H.L. Bell \& Columbus }\end{array}$ & $\begin{array}{l}\text { **Reederochloa eludens Soderstr. \& H.F. } \\
\text { Decker }\end{array}$ \\
\hline $\begin{array}{l}\text { ii) Elymus arizonicus (Scribn. \& J.G. Sm.) } \\
\text { Gould }\end{array}$ & $\begin{array}{l}\text { Agropyron spicatum (Pursh) Scribn. var. } \\
\text { arizonicum (Scribn. \& J.G. Sm.) M.E. } \\
\text { Jones }\end{array}$ \\
\hline iii) Elymus elymoides (Raf.) Swezey & **Sitanion hystrix (Nutt.) J.G. Sm. \\
\hline ii) Elymus repens (L.) Gould & **Agropyron repens (L.) P. Beauv. \\
\hline
\end{tabular}


Table 1. Continuation.

\begin{tabular}{l} 
Accepted name \\
\hline ii) Elymus trachycaulus (Link) Gould ex \\
Shinners \\
ii)*Hopia obtusa (Kunth) Zuloaga \& \\
Morrone \\
ii) Leptochloa crinita (Lag.) P.M. Peterson \\
ii)*Megathyrsus maximus (Jacq.) B.K. \\
Simon \\
ii)*Melinis repens (Willd.) Zizka \\
ii)*Mnesithea granularis (L.) de Koning \\
\& Sosef
\end{tabular}

iii) Muhlenbergia alopecuroides (Griseb.)

P. M. Peterson \& Columbus

iii) Muhlenbergia biloba Hitchc.

iii) Muhlenbergia cenchroides (Humb. \&

Bonpl. ex Willd.) P.M. Peterson

i) Muhlenbergia emersleyi Vasey

iii) Muhlenbergia ligulata (E. Fourn.)

Scribn. \& Merr.

iii) Muhlenbergia pereilema P.M. Peterson

iii) Muhlenbergia phalaroides (Kunth)

P.M. Peterson

iii) Muhlenbergia phleoides (Kunth)

Columbus

iii) Muhlenbergia shepherdii (Vasey)

Swallen

iii) Muhlenbergia subbiflora Hitchc.

iii) Muhlenbergia tricholepis (Torr.)

Columbus

iii) Muhlenbergia uniseta (Lag.) Columbus

i) Paspalum denticulatum Trin.

ii)*Peyritschia deyeuxioides (Kunth) Finot

ii)*Peyritschia pringlei (Scribn.) S.D.

Koch

i) Setaria pumila (Poir.) Roem. \& Schult.
Old name (synonym)

Agropyron trachycaulum (Link) Malte ex

H.F. Lewis

Panicum obtusum Kunth

Chloris crinita Lag \& N. Snow

Panicum maximum Jacq.\& S.W.L. Jacobs

**Rhynchelytrum repens (Willd.) C.E. Hubb.

**Hackelochloa granularis (L.) Kuntze

**Lycurus setosus (Nutt.) Reeder

**Bealia mexicana Scribn.

**Aegopogon cenchroides Humb. \& Bonpl. ex Willd.

Muhlenbergia distans Swallen

**Chaboissaea ligulata E. Fourn.

**Pereilema crinitum J. Presl

Lycurus phalaroides Kunth

Lycurus phleoides Kunth

**Blepharoneuron shepherdii (Vasey) P.M. Peterson \& Annable

Chaboissaea subbiflora (Hitchc.) Reeder \& C. Reeder

Blepharoneuron tricholepis (Torr.) Nash

Aegopogon tenellus (DC.) Trin.

Paspalum lividum Trin. ex Schltdl.

Trisetum deyeuxioides (Kunth) Kunth

Trisetum kochianum Hern. Torres

Setaria lutescens (Weigel ex Stuntz) F.T.

Hubb. 
Table 1. Continuation.

\begin{tabular}{|c|c|}
\hline Accepted name & Old name (synonym) \\
\hline $\begin{array}{l}\text { ii) *Steinchisma cuprea (Hitchc. \& Chase) } \\
\text { W.V. Br. }\end{array}$ & Panicum cupreum Hitchc. \& Chase \\
\hline $\begin{array}{l}\text { ii) *Torreyochloa pallida var. pauciflora (J. } \\
\text { Presl) J.I. Davis }\end{array}$ & Glyceria pauciflora J. Presl \\
\hline i) Trachypogon spicatus (L. f.) Kuntze & $\begin{array}{l}\text { Trachypogon plumosus (Humb. \& Bonpl. } \\
\text { ex Willd.) Nees }\end{array}$ \\
\hline $\begin{array}{l}\text { i) Urochloa fusca (Sw.) B.F. Hansen \& } \\
\text { Wunderlin }\end{array}$ & Urochloa fasciculata Kunth \\
\hline ii)*Zuloagaea bulbosa (Kunth) Bess & Panicum bulbosum Kunth \\
\hline
\end{tabular}

Table 2. Additional taxa (species and infraspecific taxa) not reported in 2001. $i=$ New records for Durango; $\mathrm{ii}=$ Recently described species not listed in Herrera Arrieta, 2001; $\mathrm{iii}=$ Reported in Herrera Arrieta et al., 2012; iv = Reported in Herrera Arrieta \& Cortés Ortíz, 2010; v = excluded in 2001 but now confirmed to occur in the state.

\begin{tabular}{lc}
\hline Species & \\
\hline Bothriochloa palmeri (Hack.) Pilg. & iv \\
Bothriochloa perforata (Trin. ex E. Fourn.) Herter & v \\
Bothriochloa saccharoides (Sw.) Rydb. & v \\
Bothriochloa springfieldii (Gould) Parodi & iv \\
Bouteloua rigidiseta (Steud.) Hitchc. & iv \\
Cenchrus brownii Roem. \& Schult. & iv \\
Cenchrus longispinus (Hack.) Fernald & iv \\
Calamagrostis divaricata P.M. Peterson \& Soreng & ii \\
Digitaria pubiflora (Vasey) Wipff. & i \\
Dinebra panicea (Retz.) P.M. Peterson \& N. Snow & iv \\
Diplachne fusca (L.) P. Beauv. ex Roem. \& Schult. & iv \\
Elymus canadensis L. & i \\
Eragrostis barrelieri Daveau & v \\
Eragrostis curvula (Schrad.) Nees & iv \\
Eragrostis erosa Scribn. ex Beal & v \\
Eragrostis pringlei Mattei & iv \\
Eriochloa contracta Hitchc. & iv \\
Festuca arizonica Vasey & iv \\
*Hesperostipa neomexicana (Thurb.) Barkworth & v
\end{tabular}


Table 2. Continuation.

\begin{tabular}{|c|c|}
\hline \multicolumn{2}{|l|}{ Species } \\
\hline Hordeum jubatum L. & $\mathrm{v}$ \\
\hline Leptochloa pluriflora (E. Fourn.) P.M. Peterson \& N. Snow & $\mathrm{v}$ \\
\hline Luziola fluitans (Michx.) Terrell \& H. Rob. & $\mathrm{i}$ \\
\hline Muhlenbergia filiculmis Vasey & iii \\
\hline Muhlenbergia longiglumis Vasey & iv \\
\hline Muhlenbergia mucronata (Kunth) Trin. & iv \\
\hline Muhlenbergia plumbea (Trin.) Hitchc. & iv \\
\hline Muhlenbergia stricta (J. Presl) Kunth & $\mathrm{v}$ \\
\hline Muhlenbergia torreyi (Kunth) Hitchc. ex Bush & iv \\
\hline Muhlenbergia villiflora Hitchc. & $\mathrm{v}$ \\
\hline Paspalum dilatatum Poir. & $\mathrm{i}$ \\
\hline Paspalum jaliscanum Chase & iv \\
\hline Piptochaetium pringlei (Beal) Parodi & $\mathrm{v}$ \\
\hline Poa matri-occidentalis P.M. Peterson \& Soreng & ii \\
\hline Sorghastrum nudipes Nash & $\mathrm{v}$ \\
\hline Sporobolus contractus Hitchc. & $\mathrm{v}$ \\
\hline Sporobolus spiciformis Swallen & iv \\
\hline Steinchisma hians (Elliott) Nash & $\mathrm{V}$ \\
\hline Tridens albescens (Vasey) Wooton \& Standl. & iv \\
\hline Trisetum durangense Finot \& P.M. Peterson & ii \\
\hline Trisetum martha-gonzaleziae P.M. Peterson \& Finot & ii \\
\hline Trisetum palmeri Hitchc. & iv \\
\hline Trisetum spicatum (L.) K. Richt. & iv \\
\hline Urochloa discifera (E. Fourn.) Morrone \& Zuloaga & iv \\
\hline Subspecies & \\
\hline Distichlis spicata subsp. stricta (Torr.) Thorne & iv \\
\hline Dinebra panicea subsp. brachiata (Steud.) P.M. Peterson \& N. Snow & iv \\
\hline Elymus elymoides subsp. brevifolius (J.G. Sm.) Barkworth & iv \\
\hline Paspalum dilatatum Poir. subsp. dilatatum & $\mathrm{i}$ \\
\hline $\begin{array}{l}\text { Poa matri-occidentalis P.M. Peterson \& Soreng subsp. matri- } \\
\text { occidentalis }\end{array}$ & ii \\
\hline Varieties & \\
\hline Aristida schiedeana Trin. \& Rupr. var. schiedeana & iv \\
\hline Aristida ternipes var. gentilis (Henrard) Allred & iv \\
\hline
\end{tabular}


Table 2. Continuation.

\begin{tabular}{lc}
\hline Varieties & \\
\hline Aristida ternipes Cav. var. ternipes & iv \\
Bothriochloa laguroides var. laguroides (DC.) Herter & i \\
Bothriochloa saccharoides (Sw.) Rydb. var. saccharoides & iv \\
Dichanthelium sphaerocarpon (Elliott) Gould var. sphaerocarpon & iv \\
Distichlis spicata var. mexicana Beetle & iv \\
Echinochloa crusgalli (L.) P. Beauv. var. crusgalli & iv \\
Echinochloa crusgalli var. zelayensis (Kunth) Hitchc. & iv \\
Echinochloa crus-pavonis (Kunth) Schult. var. crus-pavonis & iv \\
Echinochloa muricata var. microstachya Wiegand & iv \\
Elymus canadensis L. var. canadensis & i \\
Eragrostis pectinacea var. miserrima (E. Fourn.) Reeder & iv \\
Eragrostis pectinacea (Michx.) Nees var. pectinacea & iv \\
Glyceria striata (Lam.) Hitchc. var. striata & i \\
Gouinia virgata (J. Presl) Scribn. var. virgata & iv \\
Muhlenbergia villiflora Hitchc. var. villiflora & iv \\
Oplismenus burmannii (Retz.) P. Beauv. var. burmannii & iv \\
Oplismenus burmannii var. nudicaulis (Vasey) McVaugh & iv \\
Panicum alatum Zuloaga \& Morrone var. alatum & iv \\
Vulpia myuros var. hirsuta Hack. & iv \\
\hline
\end{tabular}

Forty-three species, 5 subspecies and 22 varieties were added to the previous treatment (Table 2).

The final result is the recognition of 91 genera, 367 species, 15 subspecies, 55 varieties and 2 forms (listed in Appendix below); it is worth mentioning that four species (Digitaria pubiflora, Elymus canadensis, Luziola fluitans, and Paspalum dilatatum) are new records for Durango.

\section{ACKNOWLEDGEMENTS}

I thank the Instituto Politécnico Nacional (SIP, COFAA) for facilities and financial support carried out at IPN CIIDIR Durango, in the framework of the project 
"Pastos más comunes en Durango" SIP-20031025; and CONABIO for financial support to the projects R010 y V024. I also recognize and thank the time dedicated to the revision of this manuscript by anonymous reviewers and by S. González and P.M. Peterson, whose recommendations substantially improved the present manuscript. Many thanks are given to my colleagues for the help at the herbarium CIIDIR.

\section{LITERATURE CITED}

Beal, W. J. 1896. Grasses of North America. Henry Holt and Company, New York. 457 pp. Bell, H. L. and J. T. Columbus. 2008. Proposal for an expanded Distichlis (Poaceae: Chloridoideae): support from molecular, morphological, and anatomical characters. Syst. Bot. 33: 536-551.

Bess, E. C., A. N. Doust, G. Davidse and E. A. Kellogg. 2006. Zuloagaea, a new genus of Neotropical grass within the "bristle clade" (Poaceae: Paniceae). Syst. Bot. 31(4): 656-670.

Chase, A. and C. D. Niles. 1962. Index to grass species. G. K. Hall \& Co., Boston, USA. 3 vols.

Chemisquy, M. A., L. M. Giussani, M. A. Scataglini, E. A. Kellogg and O. Morrone. 2010. Phylogenetic studies favour the unification of Pennisetum, Cenchrus and Odontelytrum (Poaceae): a combined nuclear, plastid and morphological analysis, and nomenclatural combinations in Cenchrus. Ann. Bot.-London 106: 107-130.

Columbus, J. T. and J. P. Smith. 2010. Nomenclatural changes for some grasses in California and the Muhlenbergia clade (Poaceae). Aliso 28: 65-67.

Dávila, P., Ma. T. Mejía-Saulés, M. Gómez-Sánchez, J. Valdés-Reyna, J. J. Ortíz, C. Morín, J. Castrejón and A. Ocampo. 2006. Catálogo de gramíneas de México. Universidad Autónoma de México - Comisión Nacional para el Conocimiento y Uso de la Biodiversidad. México, D.F., México. 671 pp.

Finot, V. L., P. M. Peterson, R. J. Soreng and F. O. Zuloaga. 2004. A revision of Trisetum, Peyritschia, and Sphenopholis (Poaceae: Pooideae: Aveninae) in Mexico and Central America. Ann. Missouri Bot. Gard. 91: 1-30.

Gentry, H. S. 1957. Los pastizales de Durango, estudio ecológico, fisiográfico y florístico. Traducido por E. Hernández Xolocotzi. Instituto Mexicano de Recursos Naturales Renovables A.C. México, D.F., México. 361 pp.

Herrera Arrieta, Y. 2001. Las gramíneas de Durango. Instituto Politécnico NacionalComisión Nacional para el Conocimiento y Uso de la Biodiversidad. México, D.F., México. 478 pp.

Herrera Arrieta, Y. and A. Cortés Ortiz. 2009. Diversidad de las gramíneas de Durango. Polibotánica 28: 49-68.

Herrera Arrieta, Y. y A. Cortés Ortiz. 2010. Listado florístico y aspectos ecológicos de la familia Poaceae para Chihuahua, Durango y Zacatecas, México. J. Bot. Res. Inst. Tex. 4(2): 711-738. 
Herrera Arrieta, Y., P. M. Peterson y M. de la Cerda L. 2004. Revisión de Bouteloua (Poaceae). Instituto Politécnico Nacional- Comisión Nacional para el Conocimiento y Uso de la Biodiversidad. México, D.F., México. 290 pp.

Herrera Arrieta, Y., C. A. Silva Salas, L. Ruacho González y O. Rosales Carrillo. 2012. Nuevos registros de poáceas para el norte de México. J. Bot. Res. Inst. Tex. 6(2): 583-586.

Hitchcock, A. S. 1913. Mexican grasses in the United States National Herbarium. Contr. U.S. Natl. Herb. 17(3): 181-389.

Hitchcock, A.S. 1935. Manual of the grasses of the United States. Government Printing Office, Washingtn, D.C., USA. 1040 pp.

Hitchcock, A. S. and A. Chase. 1951. Manual of the grasses of the United States. 2nd ed. United States Department of Agriculture, Washington, D.C., USA. 1051 pp.

Judziewicz, E. J., R. J. Soreng, G. Davidse, P. M. Peterson, T. S. Filgueiras and F. O. Zuloaga. 2000. Catalogue of New World grasses (Poaceae): I. Subfamilies Anomochlooideae, Bambusoideae, Ehrhartoideae, and Pharoideae. Contr. U.S. Natl. Herb. 39: 1-128.

McVaugh, R. 1983. Gramineae. In: Anderson, W. R. (ed.). Flora Novo-Galiciana Vol 14. The University of Michigan Press. Ann Arbor, USA. 436 pp.

Peterson, P. M., R. J. Soreng and Y. Herrera Arrieta. 2006. Poa matri-occidentalis (Poaceae: Pooideae: Poeae: Poinae), a new species from Mexico. Sida 22(2): 905-914.

Peterson, P. M., R. J. Soreng and J. Valdés-Reyna. 2004. Calamagrostis coahuilensis and C. divaricata (Poaceae: Pooideae: Agrostidinae) two new species from México. Sida 21(1): 311-320.

Peterson, P. M. and J. T. Columbus. 2009. Muhlenbergia tarahumara (Poaceae: Chloridoideae: Cynodonteae: Muhlenbergiinae), a new species from Chihuahua, México. J. Bot. Res. Inst. Tex. 3(2): 527-534.

Peterson, P. M., K. Romaschenko and G. Johnson. 2010. A phylogeny and classification of the Muhlenbergiinae (Poaceae: Chloridoideae: Cynodonteae) based on plastid and nuclear DNA sequences. Amer. J. Bot. 97(9): 1532-1554.

Peterson, P. M., K. Romaschenko, N. Snow and G. Johnson. 2012. A molecular phylogeny and classification of Leptochloa (Poaceae: Chloridoideae: Chlorideae) sensu lato and related genera. Ann. Bot.-London. 109: 1317-1329.

Peterson, P. M., R. J. Soreng, G. Davidse, T. S. Filgueiras, F. O. Zuloaga and E. J. Judziewicz. 2001. Catalogue of New World grasses (Poaceae): II. Subfamily Chloridoideae. Contr. U.S. Natl. Herb. 41: 1-255.

Soreng, R.J., G. Davidse, P.M. Peterson, F.O. Zuloaga, E.J. Judziewicz, T.S. Filgueiras, and O. Morrone. 2013. Catalogue of New World grasses (Poaceae). http://www.tropicos. org/Project/CNWG, accessed 9 May 2013.

Soreng, R. J., P. M. Peterson, G. Davidse, E. J. Judziewicz, F. O. Zuloaga, T. S. Filgueiras and O. Morrone. 2003. Catalogue of New World grasses (Poaceae): IV. Subfamily Pooideae. Contr. U.S. Natl. Herb. 48: 1-730.

Valdés-Reyna, J., F. O. Zuloaga, O. Morrone y L. Aragón. 2009. El género Panicum (Poaceae: Panoicoideae) en el noreste de México. Bol. Soc. Bot. Méx. 84: 59-82.

Zuloaga, F. O., L. M. Guissani and O. Morrone. 2007. Hopia, a new monotypic genus segregated from Panicum. Taxon 56: 145-156. 
Zuloaga, F. O., O. Morrone, G. Davidse, T. S. Filgueiras, P. M. Peterson, R. J. Soreng and E. Judziewicz. 2003. Catalogue of New World grasses (Poaceae): III. Subfamilies Panicoideae, Aristidoideae, Arundinoideae, and Danthonioideae. Contr. U.S. Natl. Herb. 46: 1-662. 


\section{APPENDIX}

\section{Updated list of Poaceae in Durango, Mexico}

\begin{tabular}{|c|c|}
\hline Achnatherum eminens (Cav.) Barkworth & B. palmeri (Hack.) Pilg. \\
\hline Agrostis exarata Trin. & B. perforata (Trin. ex E. Fourn.) Herter \\
\hline A. liebmannii (E. Fourn.) Hitchc. & B. saccharoides (Sw.) Rydb. var. \\
\hline A. perennans (Walter) Tuck. & saccharoides \\
\hline A. rosei Scribn. \& Merr. & B. springfieldii (Gould) Parodi \\
\hline A. scabra Willd. & B. wrightii (Hack.) Henrard \\
\hline A. tolucensis Kunth & Bouteloua aristidoides (Kunth) Griseb. var. \\
\hline Andropogon gerardii Vitman & aristidoides \\
\hline A. glomeratus var. pumilus (Vasey) Vasey & B. barbata Lag. var. barbata \\
\hline A. pringlei Scribn. \& Merr. & B. chondrosioides (Kunth) Benth. \& S. \\
\hline Anthoxanthum odoratum $\mathrm{L}$. & Watson \\
\hline Aristida adscensionis L. & B. curtipendula var. caespitosa Gould \& \\
\hline A. appressa Vasey & Kapadia \\
\hline A. arizonica Vasey & B. curtipendula (Michx.) Torr. var. \\
\hline A. divaricata Humb. \& Bonpl. ex Willd. & curtipendula \\
\hline A. eludens Allred \& Valdés-Reyna & B. curtipendula var. tenuis Gould \& \\
\hline A. gibbosa (Nees) Kunth & Kapadia \\
\hline A. havardii Vasey & B. dactyloides (Nutt.) Columbus \\
\hline A. laxa Cav. var. laxa & B. gracilis (Kunth) Lag. ex Griffiths \\
\hline A. pansa Wooton \& Standl. fo. pansa & B. hirsuta var. glandulosa (Cerv.) Gould \\
\hline A. purpurea var. nealleyi (Vasey) Allred & B. hirsuta Lag. var. hirsuta \\
\hline A. purpurea Nutt. var. purpurea & B. parryi var. gentryi (Gould) Gould \\
\hline $\begin{array}{l}\text { A. schiedeana var. orcuttiana (Vasey) Allred } \\
\text { \& Valdés-Reyna }\end{array}$ & $\begin{array}{l}\text { B. parryi (E. Fourn.) Griffiths var. parryi } \\
\text { B. polymorpha (E. Fourn.) Columbus }\end{array}$ \\
\hline A. schiedeana Trin. \& Rupr. var. schiedeana & B. radicosa (E. Fourn.) Griffiths \\
\hline A. scribneriana Hitchc. & B. ramosa Scribn. ex Vasey \\
\hline A. spanospicula Allred, Valdés-Reyna \& & B. reederorum Columbus \\
\hline Sánchez-Ken & B. repens (Kunth) Scribn. \\
\hline A. ternipes var. gentilis (Henrard) Allred & B. rigidiseta (Steud.) Hitchc. \\
\hline A. ternipes Cav. var. ternipes & B. scorpioides Lag. \\
\hline Arundinella hispida (Humb. \& Bonpl. ex & B. simplex Lag. \\
\hline Willd.) Kuntze & B. uniflora var. coahuilensis Gould \& \\
\hline Arundo donax L. fo. donax & Kapadia \\
\hline Avena fatua $\mathrm{L}$. & Brachypodium mexicanum (Roem. \& \\
\hline A. sativa $\mathrm{L}$. & Schult.) Link var. mexicanum \\
\hline Axonopus mexicanus G.A. Black & Bromus anomalus Rupr. ex E. Fourn. \\
\hline Bothriochloa alta (Hitchc.) Henrard & B. carinatus var. californicus Shear \\
\hline B. barbinodis (Lag.) Herter & B. catharticus Vahl var. catharticus \\
\hline B. laguroides (DC.) Herter var. laguroides & B. mucroglumis Wagon \\
\hline
\end{tabular}


Appendix. Continuation.

B. porteri (J.M. Coult.) Nash

$B$. richardsonii Link

B. thysanoglottis Soderstr. \& Beaman

Calamagrostis divaricata P.M. Peterson \& Soreng

C. pringlei Scribn. ex Beal

C. valida Sohns

Cenchrus brownii Roem. \& Schult.

C. ciliaris L.

C. clandestinus (Hochst. ex Chiov.) Morrone

C. echinatus L.

C. longispinus (Hack.) Fernald

C. michoacanus H.F. Gut. \& Morrone

C. multiflorus J. Presl

C. myosuroides Kunth var. myosuroides

C. spinifex Cav.

C. villosus (R. Br. ex Fresen.) Kuntze

Chascolytrum subaristatum (Lam.) Desv.

Chloris gayana Kunth

C. rufescens Lag.

C. submutica Kunth

C. virgata $\mathrm{Sw}$.

Cortaderia selloana (Schult. \& Schult. f.) Asch. \& Graebn.

Cottea pappophoroides Kunth

Cynodon dactylon (L.) Pers.

C. plectostachyus (K. Schum.) Pilg.

Dactyloctenium aegyptium (L.) Willd.

Dasyochloa pulchella (Kunth) Willd. ex Rydb.

Deschampsia flexuosa (L.) Trin.

Dichanthelium sphaerocarpon (Elliott)

Gould var. sphaerocarpon

Digitaria californica (Benth.) Henrard var. californica

D. ciliaris (Retz.) Koeler

D. filiformis (L.) Koeler

D. insularis (L.) Fedde

D. panicea (Sw.) Urb.

D. pubiflora (Vasey) Wipff.
D. sanguinalis (L.) Scop.

D. ternata (Hochst. ex A. Rich.) Stapf

Dinebra panicea ssp. brachiata (Steud.)

P.M. Peterson \& N. Snow

D. panicea ssp. mucronata (Michx.) P.M.

Peterson \& N. Snow

Diplachne fusca ssp. fascicularis (Lam.)

P.M. Peterson \& N. Snow

D. fusca ssp. uninervia (J. Presl) P.M.

Peterson \& N. Snow

Disakisperma dubium (Kunth) P.M.

Peterson \& N. Snow

Distichlis eludens (Soderstr. \& H.F. Decker)

H.L. Bell \& Columbus

D. spicata var. mexicana Beetle

D. spicata ssp. stricta (Torr.) Thorne

Echinochloa colona (L.) Link

E. crusgalli (L.) P. Beauv. var. crusgalli

E. crusgalli var. zelayensis (Kunth) Hitchc.

E. crus-pavonis (Kunth) Schult. var. cruspavonis

E. holciformis (Kunth) Chase

E. muricata var. microstachya Wiegand

E. oplismenoides (E. Fourn.) Hitchc.

E. polystachya (Kunth) Hitchc. var. polystachya

Eleusine indica (L.) Gaertn.

E. multiflora Hochst. ex A. Rich.

Elionurus barbiculmis Hack.

E. tripsacoides Humb. \& Bonpl. ex Willd.

Elymus arizonicus (Scribn. \& J.G. Sm.)

Gould

E. canadensis L. var. canadensis

E. elymoides ssp. brevifolius (J.G. Sm.)

Barkworth

E. repens (L.) Gould ssp. repens

E. riparius Wiegand

E. trachycaulus (Link) Gould ex Shinners

ssp. trachycaulus

Enneapogon desvauxii P. Beauv.

Eragrostis barrelieri Daveau 
Appendix. Continuation.

E. cilianensis (All.) Vignolo ex Janch.

E. ciliaris (L.) R. Br. var. ciliaris

E. curvula (Schrad.) Nees

E. erosa Scribn. ex Beal

E. glandulosa L.H. Harv.

E. intermedia Hitchc. var. intermedia

E. lehmanniana Nees

E. lugens Nees

E. maypurensis (Kunth) Steud.

E. mexicana (Hornem.) Link ssp. mexicana

E. palmeri S. Watson

E. pectinacea var. miserrima (E. Fourn.)

Reeder

E. pectinacea (Michx.) Nees var. pectinacea

E. pilosa (L.) P. Beauv.

E. pringlei Mattei

E. viscosa (Retz.) Trin.

Eriochloa acuminata (J. Presl) Kunth var. acuminata

E. aristata Vasey var. aristata

E. contracta Hitchc.

E. lemmonii Vasey \& Scribn.

E. nelsonii Scribn. \& J.G. Sm.

Erioneuron avenaceum (Kunth) Tateoka var. avenaceum

E. nealleyi (Vasey) Tateoka

Festuca amplissima Rupr.

F. arizonica Vasey

F. breviglumis Swallen

$F$. pringlei St. Yves

F. rosei Piper

F. tolucensis Kunth

Glyceria fluitans (L.) R. Br.

G. striata (Lam.) Hitchc. var. striata

Gouinia virgata (J. Presl) Scribn. var. virgata

Hesperostipa neomexicana (Thurb.)

Barkworth

Heteropogon contortus (L.) P. Beauv. ex

Roem. \& Schult.

H. melanocarpus (Elliott) Benth.
Hilaria belangeri (Steud.) Nash var. belangeri

H. cenchroides Kunth

H. mutica (Buckley) Benth.

H. swallenii Cory

Hopia obtusa (Kunth) Zuloaga \& Morrone

Hordeum jubatum L.

Hyparrhenia rufa (Nees) Stapf

Koeleria pyramidata (Lam.) P. Beauv.

Lasiacis nigra Davidse

L. procerrima (Hack.) Hitchc.

L. ruscifolia (Kunth) Hitchc.

Leersia hexandra Sw.

Leptochloa crinita (Lag.) P.M. Peterson \& N. Snow

L. pluriflora (E. Fourn.) P.M. Peterson \& N. Snow

Lolium multiflorum Lam.

L. perenne $\mathrm{L}$.

Luziola fluitans (Michx.) Terrell \& H. Rob.

Megathyrsus maximus (Jacq.) B.K. Simon

\& S.W.L. Jacobs

Melinis repens (Willd.) Zizka

Microchloa kunthii Desv.

Mnesithea granularis (L.) de Koning \&

Sosef

Muhlenbergia alamosae Vasey

M. alopecuroides (Griseb.) P.M. Peterson \&

Columbus

M. annua (Vasey) Swallen

M. arenicola Buckley

$M$. arizonica Scribn.

M. asperifolia (Nees \& Meyen ex Trin.)

Parodi

M. biloba Hitchc.

$M$. brevifolia Scribn. ex Beal

M. brevis C.O. Goodd.

$M$. brevivaginata Swallen

M. cenchroides (Humb. \& Bonpl. ex Willd.)

P.M. Peterson

M. ciliata (Kunth) Trin. 
Appendix. Continuation.

M. crispiseta Hitchc.

M. depauperata Scribn.

M. diversiglumis Trin.

M. dubia E. Fourn.

M. dumosa Scribn. ex Beal

M. durangensis Y. Herrera

M. eludens C. Reeder

M. emersleyi Vasey

M. eriophylla Swallen

M. filiculmis Vasey

M. filiformis (Thurb. ex S. Watson) Rydb.

M. flavida Vasey

M. flaviseta Scribn.

M. fragilis Swallen

M. gigantea (E. Fourn.) Hitchc.

M. glauca (Nees) B.D. Jacks.

M. implicata (Kunth) Trin.

M. ligulata (E. Fourn.) Scribn. \& Merr.

M. longiglumis Vasey

M. longiligula Hitchc.

M. macroura (Kunth) Hitchc.

M. michisensis Y. Herrera \& P.M. Peterson

M. microsperma (DC.) Kunth

M. minutissima (Steud.) Swallen

M. montana (Nutt.) Hitchc.

M. mucronata (Kunth) Trin.

M. palmeri Vasey

M. pauciflora Buckley

M. pectinata C.O. Goodd.

M. pereilema P.M. Peterson

M. peruviana (P. Beauv.) Steud.

M. phalaroides (Kunth) P.M. Peterson

M. phleoides (Kunth) Columbus

M. plumbea (Trin.) Hitchc.

M. polycaulis Scribn.

M. porteri Scribn. ex Beal

M. pubescens (Kunth) Hitchc.

M. quadridentata (Kunth) Trin.

M. ramulosa (Kunth) Swallen

$M$. reederorum Soderstr.

M. repens (J. Presl) Hitchc.
M. richardsonis (Trin.) Rydb.

$M$. rigens (Benth.) Hitchc.

M. rigida (Kunth) Trin.

M. robusta (E. Fourn.) Hitchc.

M. scoparia Vasey

M. setifolia Vasey

M. shepherdii (Vasey) Swallen

M. speciosa Vasey

M. stricta (J. Presl) Kunth

M. strictior Scribn. ex Beal

M. subaristata Swallen

M. subbiflora Hitchc.

M. tenella (Kunth) Trin.

M. tenuifolia (Kunth) Kunth

M. texana Buckley

M. torreyi (Kunth) Hitchc. ex Bush

M. tricholepis (Torr.) Columbus

M. uniseta (Lag.) Columbus

M. utilis (Torr.) Hitchc.

M. vaginata Swallen

M. villiflora Hitchc. var. villiflora

M. virescens (Kunth) Trin.

M. virletii (E. Fourn.) Soderstr.

Nassella leucotricha (Trin. \& Rupr.) R.W. Pohl

N. mucronata (Kunth) R.W. Pohl

N. tenuissima (Trin.) Barkworth

Oplismenus burmannii (Retz.) P. Beauv. var. burmannii

O. burmannii var. nudicaulis (Vasey)

McVaugh

O. compositus (L.) P. Beauv.

Otatea acuminata ssp. aztecorum (McClure \& E.W. Sm.) R. Guzmán, Anaya \& Santana $O$. fimbriata Soderstr.

Panicum alatum Zuloaga \& Morrone var. alatum

P. antidotale Retz.

$P$. decolorans Kunth

P. dichotomiflorum Michx.

P. hallii Vasey var. hallii 
Appendix. Continuation.

\begin{tabular}{|c|c|}
\hline $\begin{array}{l}\text { P. hirticaule var. verrucosum Zuloaga \& } \\
\text { Morrone }\end{array}$ & $\begin{array}{l}\text { Schizachyrium cirratum (Hack.) Wooton \& } \\
\text { Standl. }\end{array}$ \\
\hline P. lepidulum Hitchc. \& Chase & S. sanguineum (Retz.) Alston \\
\hline P. parcum Hitchc. \& Chase & S. scoparium var. neomexicanum (Nash) \\
\hline P. plenum Hitchc. \& Chase & Hitchc. \\
\hline P. stramineum Hitchc. \& Chase & S. tenerum Nees \\
\hline P. trichoides Sw. & Scleropogon brevifolius Phil. \\
\hline P. vaseyanum Scribn. ex Beal & Setaria adhaerens (Forssk.) Chiov. \\
\hline Pappophorum bicolor E. Fourn. & S. grisebachii E. Fourn. \\
\hline P. vaginatum Buckley & S. latifolia (Scribn.) R.A.W. Herrm. \\
\hline Paspalum conjugatum P.J. Bergius & S. leucopila (Scribn. \& Merr.) K. Schum. \\
\hline P. convexum Humb. \& Bonpl. ex Flüggé & S. liebmannii E. Fourn. \\
\hline P. crinitum Chase & S. macrostachya Kunth \\
\hline P. denticulatum Trin. & S. parviflora (Poir) Kerguélen \\
\hline P. dilatatum Poir. ssp. dilatatum & S. pumila (Poir.) Roem. \& Schult. \\
\hline P. distichum $\mathrm{L}$. & S. scheelei (Steud.) Hitchc. \\
\hline P. humboldtianum Flüggé & S. verticillata (L.) P. Beauv. \\
\hline P. jaliscanum Chase & S. viridis (L.) P. Beauv. \\
\hline P. notatum Flüggé & Sorghastrum nudipes Nash \\
\hline P. plicatulum Michx. & S. nutans (L.) Nash \\
\hline P. prostratum Scribn. \& Merr. & Sorghum bicolor (L.) Moench \\
\hline P. pubiflorum Rupr. ex E. Fourn. & S. halepense (L.) Pers. \\
\hline Peyritschia deyeuxioides (Kunth) Finot & Sphenopholis obtusata (Michx.) Scribn. \\
\hline P. pringlei (Scribn.) S.D. Koch & Sporobolus airoides (Torr.) Torr. var. \\
\hline Phalaris canariensis $\mathrm{L}$. & airoides \\
\hline Phragmites australis (Cav.) Trin. ex Steud. & S. atrovirens (Kunth) Kunth \\
\hline Piptochaetium brevicalyx ssp. flexиosum & S. coahuilensis Valdés-Reyna \\
\hline Barkworth & S. contractus Hitchc. \\
\hline P. fimbriatum (Kunth) Hitchc. & S. cryptandrus (Torr.) A. Gray \\
\hline P. pringlei (Beal) Parodi & S. indicus (L.) R. Br. var. indicus \\
\hline P. virescens (Kunth) Parodi & S. macrospermus Scribn. ex Beal \\
\hline Роа аппиа $\mathrm{L}$. & S. palmeri Scribn. \\
\hline $\begin{array}{l}\text { P. matri-occidentalis P.M. Peterson \& } \\
\text { Soreng ssp. matri-occidentalis }\end{array}$ & $\begin{array}{l}\text { S. pyramidatus (Lam.) Hitchc. } \\
\text { S. spiciformis Swallen }\end{array}$ \\
\hline P. strictiramea Hitchc. & S. trichodes Hitchc. \\
\hline Polypogon elongatus Kunth & S. wrightii Munro ex Scribn. \\
\hline P. interruptus Kunth & Steinchisma cuprea (Hitchc. \& Chase) W.V. \\
\hline P. monspeliensis (L.) Desf. & $\mathrm{Br}$. \\
\hline P. viridis (Gouan) Breistr. & S. hians (Elliott) Nash \\
\hline $\begin{array}{l}\text { Rhipidocladum racemiflorum (Steud.) } \\
\text { McClure }\end{array}$ & $\begin{array}{l}\text { Torreyochloa pallida var. pauciflora (J. } \\
\text { Presl) J.I. Davis }\end{array}$ \\
\hline
\end{tabular}


Appendix. Continuation.

Trachypogon spicatus (L. f.) Kuntze

Tragus berteronianus Schult.

Tridens albescens (Vasey) Wooton \& Standl.

T. muticus (Torr.) Nash

Tripogon spicatus (Nees) Ekman

Tripsacum dactyloides (L.) L. var. dactyloides

T. lanceolatum Rupr. ex E. Fourn.

T. pilosum Scribn. \& Merr.

T. zopilotense Hern.-Xol. \& Randolph

Trisetum durangense Finot \& P.M. Peterson

T. filifolium var. aristatum Scribn. ex Beal

T. irazuense (Kuntze) Hitchc.

T. martha-gonzaleziae P.M. Peterson \& Finot

T. palmeri Hitchc.
T. spicatum (L.) K. Richt.

T. viride (Kunth) Kunth

T. virletii E. Fourn.

Tristachya laxa Scribn. \& Merr.

Triticum aestivum $\mathrm{L}$.

Urochloa arizonica (Scribn. \& Merr.)

Morrone \& Zuloaga

$U$. discifera (E. Fourn.) Morrone \& Zuloaga

U. fusca (Sw.) B.F. Hansen \& Wunderlin

U. meziana (Hitchc.) Morrone \& Zuloaga

U. plantaginea (Link) R.D. Webster

Vulpia microstachys (Nutt.) Munro

V. myuros var. hirsuta Hack.

V. octoflora (Walter) Rydb.

Zea mays L. ssp. mays

Z. mays ssp. mexicana (Schrad.) H.H. Iltis

Zuloagaea bulbosa (Kunth) Bess 